Received: 2017.02.07

Accepted: 2017.02 .26

Published: 2017.11 .17

\title{
Lumbar Opening Pressure and Radiologic Scoring in Idiopathic Intracranial Hypertension: Is There Any Correlation?
}

Authors' Contribution:
A Study Design
B Data Collection
C Statistical Analysis
D Data Interpretation
E Manuscript Preparation
F Literature Search
G Funds Collection

\author{
Sedat Alpaslan Tuncel ${ }^{1 \mathrm{ABCDEF}}$, Erdem Yılmaz ${ }^{1 \mathrm{CDE}}$, Bekir Çağlı ${ }^{1 \mathrm{ABDDE}}$, Aslan Tekataş2 ${ }^{2 \mathrm{ABCD}}$, \\ Yahya Çelik ${ }^{2 \mathrm{ABD}}$, Mehmet Ercüment Ünlü1 ${ }^{1 \mathrm{ACEF}}$
}

${ }^{1}$ Department of Radiology, Balkan Campus, Trakya University Faculty of Medicine, Edirne, Turkey

2 Department of Neurology, Balkan Campus, Trakya University Faculty of Medicine, Edirne, Turkey

Author's address: Sedat Alpaslan Tuncel, Department of Radiology, Trakya University, School of Medicine, 22000 Edirne, Turkey, e-mail: deusedattuncel@yahoo.com
Material/Methods:

Results:

Conclusions:

MeSH Keywords:

PDF file:

\section{Summary}

To investigate correlation between lumbar opening pressure (LOP) and radiological scores based on cranial MRI and contrast-enhanced MR venography in patients with idiopathic intracranial hypertension (IIH).

Patients with IIH who underwent brain MRI and contrast-enhanced MR venography before measurement of LOP between 2010-2014 were evaluated retrospectively. Three experienced radiologists (blinded to LOP values) evaluated a total of 51 patients. They reached a consensus on the presence or absence of 6 radiological findings identified in the literature as characteristic for IIH: empty sella, perioptic dilation, optical tortuosity, flattening of the posterior globe, swelling of the optic disc, and bilateral transverse sinus stenosis. The radiological score was obtained by giving 1 point for the presence of each finding, with the highest possible score of 6 points. The correlation between the calculated radiological scores and LOP was evaluated.

There was no significant correlation between LOP and radiological scores $(r=0.095 ; p=0.525$, Spearman's rank coefficient). Similarly, no significant correlation was detected between LOP and each of the radiological findings (partial empty sella $[p=0.137]$, perioptic dilation $[p=0.265]$, optical tortuosity $[p=0.948]$, flattening of the posterior globe $[p=0.491]$, swelling of the optic disc $[p=0.881]$, and bilateral dural sinus stenosis [p=0.837], Mann-Whitney U test).

There was no significant correlation between LOP and reliable radiological features of IIH.

\section{Headache • Magnetic Resonance Imaging • Pseudotumor Cerebri}

http://www.polradiol.com/abstract/index/idArt/903662

\section{Background}

Idiopathic intracranial hypertension (IIH) is a neuropthalmological disease that was previously described as serous meningitis, pseudotumor cerebrum, or benign intracranial hypertension. Classically, it has been characterized by headaches and impaired vision. Although IIH can be seen in both males and females of any age, it is more common in obese women of the reproductive age. IIH used to be described as benign, but this has been abandoned due to negative effects of headaches on everyday life and risk of permanent vision loss. The description of pseudotumor cerebri has been applied only in the presence of secondary causes and/or when those causes co-occur with the disease [1].

It is important to exclude intracranial causes of IIH, such as tumors, vascular anomalies, or sinus thrombosis that could have similar symptoms as IIH. Therefore, radiological imaging is critical. In the current diagnostic criteria published in 2013, a possible diagnosis of IIH was added in patients without papilledema. The diagnosis of possible IIH requires at least 3 of the following 4 magnetic resonance imaging (MRI) and magnetic resonance venography (MRV) 


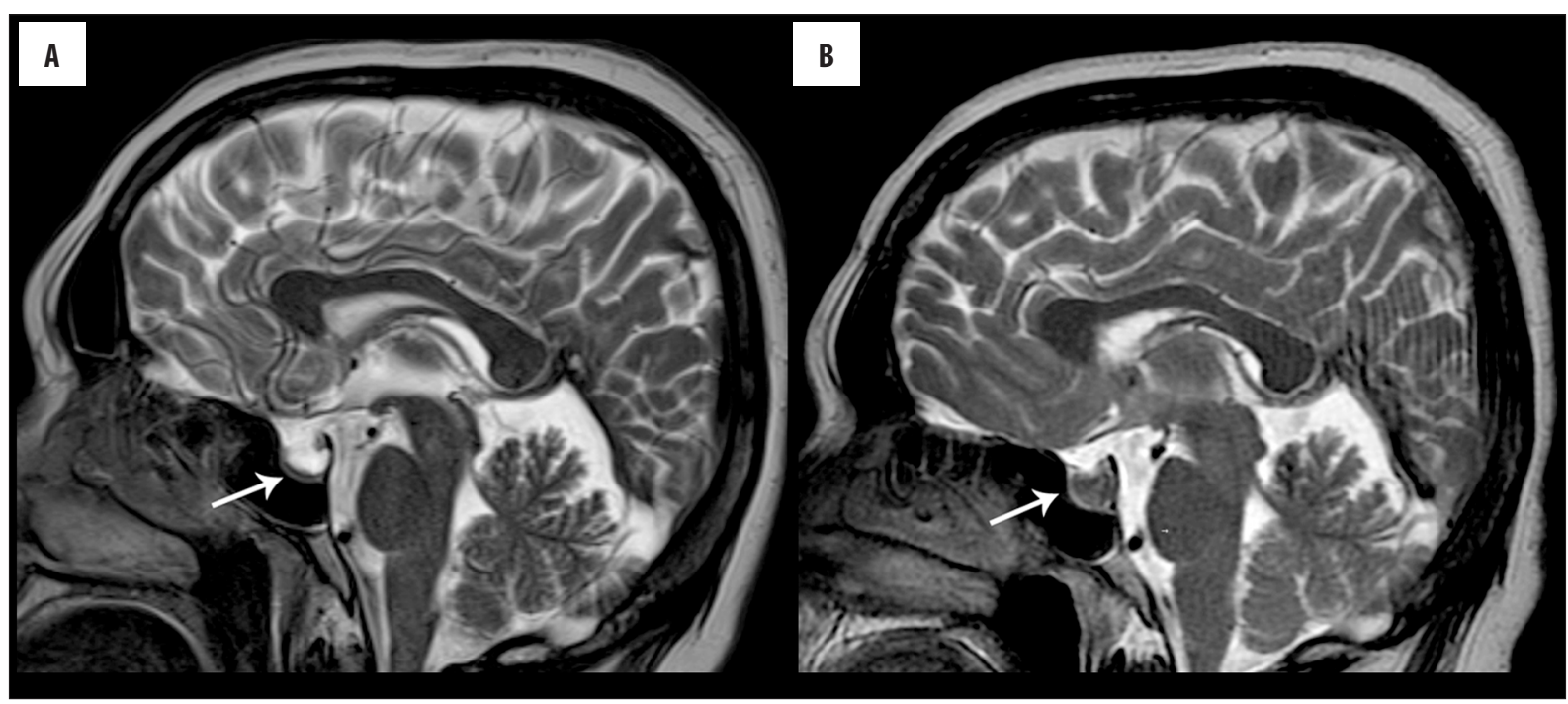

Figure 1. Mid-sagittal T2-weighted images in a 43-year-old woman with IIH (lumbar opening pressure: $60 \mathrm{~cm} \mathrm{H}_{2} \mathrm{O}$ ). (A) "Empty sella" in the pretreatment image. (B) Normal height of the pituitary gland was observed after treatment.

findings: empty sella, perioptic dilatation, flattening of the posterior globe, and bilateral transverse sinus stenosis [2]. Although several theories have been put forward with regard to the etiopathogenesis of IIH, a definitive consensus has not yet been reached. Obesity, hormonal factors, certain medications, autoimmunity, anemia, biochemical pathologies, and even genetic factors and diseases associated with inbreeding have been reported to co-occur with IIH $[1,3]$.

Currently, the absence of papilledema or normal lumbar opening pressure (LOP) does not definitively exclude IIH. Therefore, radiological imaging and in particular contrastenhanced MRV intended for dural sinuses have gained utmost importance. In our study, we aimed to investigate correlation between LOP and radiological scores derived from cranial MRI and contrast-enhanced MRV findings in patients diagnosed with IIH. To our knowledge, this is the first study than employed contrast-enhanced MRV, which is more reliable than other methods in showing transverse sinus stenosis.

\section{Material and Methods}

The local ethics committee approved this retrospectively designed study. All patients gave informed consent prior to inclusion. Medical files of patients with a preliminary diagnosis of pseudotumor cerebri or IIH and who underwent routine brain MRI and contrast-enhanced 3D MRV in our clinic between January 2010 and December 2014 were obtained from our radiology archive (Radiologist Workstation - IDS7 ${ }^{\mathrm{TM}} / \mathrm{DX}$, Sectra Medical Systems, Sweden). Pediatric patients, patients with infectious, neoplastic, vascular (aneurysm, dural sinus thrombosis, malformations), and structural abnormalities in the brain, patients with a history of cranial surgery, patients with inadequate imaging, and patients with abnormalities in the cerebrospinal fluid were excluded.

MR imaging was performed with a $1.5 \mathrm{~T}$ MR scanner (Signa HDx; GE Healthcare, Milwaukee, Wisconsin) and a standard head coil (8-channel). Routine coronal, axial, and sagittal $\mathrm{T} 2$-weighted images $(\mathrm{TR}=3440-4100 \mathrm{~ms}$; $\mathrm{TE}=84-88 \mathrm{~ms}$; field of view $22-23 \mathrm{~cm}$; slice thickness 5-5.5 mm; interslice gap 0.5-1 mm; echo train length, 16; matrix, 352 $\times 256$; number of average, 0.5-2) and contrastenhanced 3D MRV ( $\mathrm{TR}=4984 \mathrm{~ms}, \mathrm{TE}=1756 \mathrm{~ms}$; field of view $23 \mathrm{~cm}$; slice thickness $1.4 \mathrm{~mm}$; number of average 1 ; echo train length 1 ; matrix $320 \times 320$; flip angle $30^{\circ}$ ) source, and maximum intensity projection (MIP) images were evaluated.

Three neuroradiologists (SAT, BC, MEU) with 7-14 years of experience and blinded to LOP values evaluated the radiological images in consensus. One point was given for each of the following findings: empty sella or partial empty sella (very thin or no pituitary gland in the sella cavity; Figure 1), tortuous optic nerve (twisting of the optic nerve in vertical or horizontal plane; Figure 2), perioptic dilatation (more than 2-mm expansion of the optic nerve sheath), flattening of the posterior globe (convexity loss and flattening in the optic nerve-globe junction), swelling of the optic disc (intraocular protrusion of the optic nerve), and bilateral transverse sinus stenosis (Figure 3 ). The resulting radiological score was the sum of all findings, with a maximum possible score of 6 points. After 24-72 hours following MR examinations, the patients underwent lumbar puncture (21-gauge spinal needle) in the lateral decubitus position.

The statistical analysis was performed using the Statistical Package for the Social Sciences, version 22.0 (SPSS, Chicago, Illinois). Correlation between LOP and radiological findings, either combined (radiological scores) or individual, was investigated with Spearman's rank correlation coefficient and the Mann-Whitney U test, respectively.

\section{Results}

Eighty-five patients who underwent cranial MRI and MRV due to a preliminary diagnosis of pseudotumor cerebri or $\mathrm{IIH}$ were found in our radiology imaging archive. Among these patients, 27 cases were excluded from the study (1 


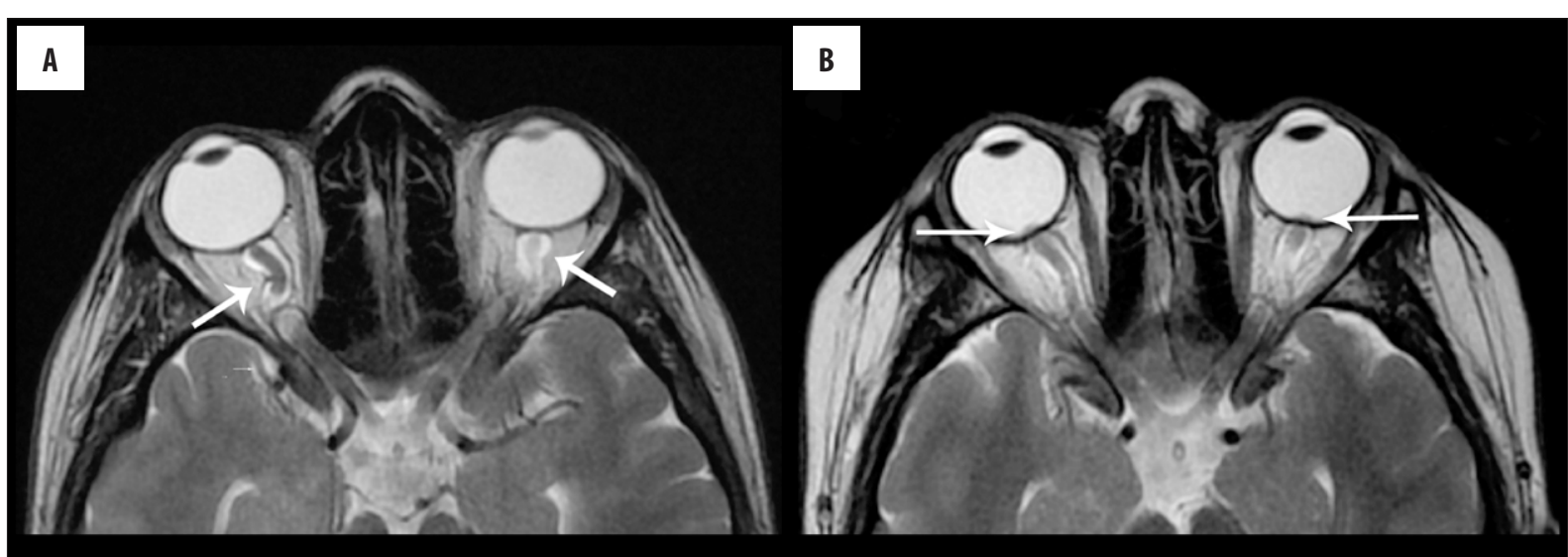

Figure 2. Orbital findings in patients with IIH on axial T2-weighted images. (A) Perioptic enlargement and optic nerve horizontal tortuosity (arrows) are demonstrated in a 38-year-old woman (lumbar opening pressure: $34 \mathrm{~cm} \mathrm{H}_{2} \mathrm{O}$ ). (B) Posterior globe flattening and swelling of the optic disc (arrows) in an 18-year-old woman (lumbar opening pressure: $27 \mathrm{~cm} \mathrm{H}_{2} \mathrm{O}$ ).

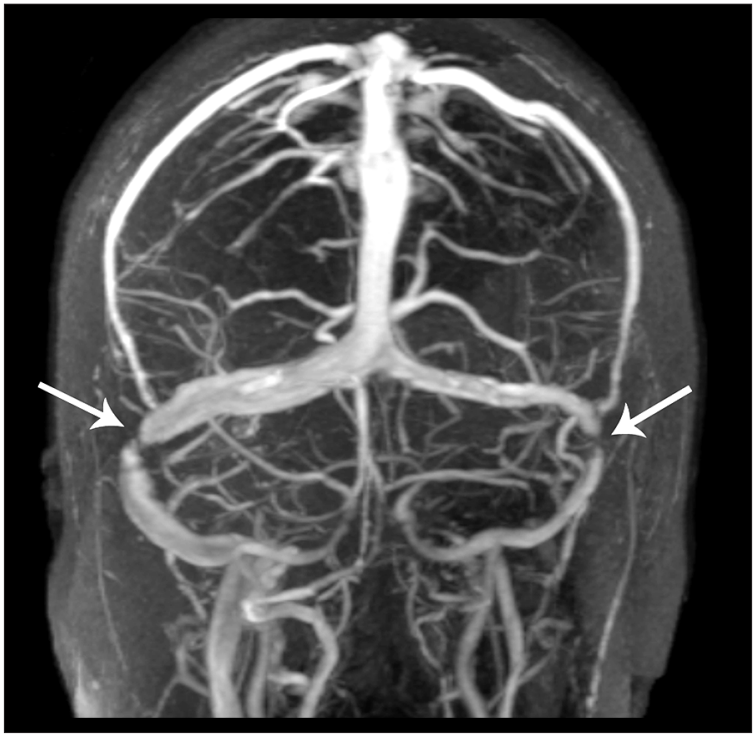

Figure 3. Bilateral focal transverse sinus stenosis (arrows) demonstrated by contrast-enhanced 3D MR venography in a 23 year-old woman (lumbar opening pressure: $55 \mathrm{~cm} \mathrm{H}_{2} 0$ ).

pediatric case, 2 aneurysms, 7 malignancies (acute myeloid leukemia, lung cancer, operated meningioma), 3 with a history of sinus thrombosis, 1 multiple sclerosis, 1 pituitary adenoma, 2 cases of epilepsy, and 10 cases with inadequate imaging). Clinical records of 7 patients were missing. Therefore, a total of 51 patients ( 45 male and 6 female) with complete imaging data and papilledema detected on fundoscopy were enrolled in the study. Four patients refused to undergo lumbar puncture. The mean age of patients was 38.63 years (range 18-63). The mean body mass index (BMI) was $30.5 \pm 7.3 \mathrm{~kg} / \mathrm{m}^{2}$ (range 17-48).

There was no significant correlation between LOP values and radiological scores $(\mathrm{r}=0.095 ; \mathrm{p}=0.525$, Spearman's rank correlation coefficient). Moreover, there was no significant correlation between LOP values and individual radiological findings (empty sella $[p=0.137]$, perioptic dilation $[p=0.265]$, optical tortuosity $[p=0.948]$, flattening of the posterior globe $[p=0.491]$, swelling of the optic disc

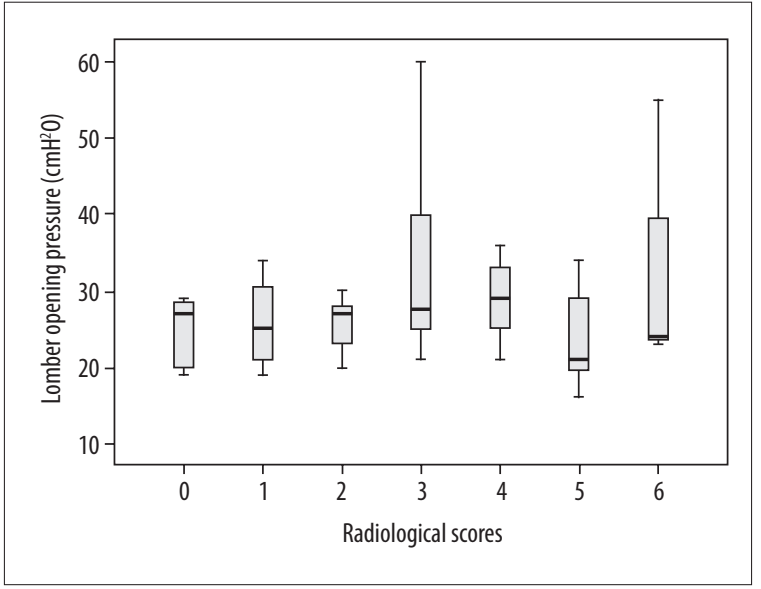

Figure 4. Comparison of the lumbar opening pressure distribution with radiographic scores (score 0 - no finding, score 1 - 1 finding, and score $6-6$ findings determined by MRI).

$[p=0.881]$, bilateral dural sinus stenosis $[p=0.837]$, MannWhitney $U$ test). The relationship between radiological scores and LOP is shown in Figure 4. The incidence of MR findings was as follows: empty sella, $51.0 \%$ (26/51); perioptic dilation, $68.0 \%$ (35/51); optical tortuosity, 56.0\% (29/51); flattening of the posterior globe, $31.0 \%$ (16/51); swelling of the optic disc, $17.0 \%$ (9/51); and bilateral transverse sinus stenosis, $41.0 \%$ (21/51). Moreover, transverse sinus hypoplasia was seen in $21.0 \%(11 / 51)$ of patients, and unilateral transverse sinus stenosis was seen in $19.0 \%(10 / 51)$ of patients.

Concomitant iron-deficiency anemia, polycystic ovary syndrome, systemic lupus erythematosus, and use of oral contraceptives were present in 10,2, 1, and 4 patients, respectively; thus, these patients can be diagnosed with pseudotumor cerebri.

\section{Discussion}

IIH used to be defined with clinical and laboratory findings. Currently, rapid advances in radiologic imaging have contributed to a more reliable diagnosis. Since clinical 
diagnosis is usually made by exclusion, evaluation of radiological findings is crucial. Moreover, some imaging findings defined in the last 20 years can support, according to the latest diagnostic criteria, the diagnosis of IIH in clinically suspicious cases; e.g., patients without papilledema or normal LOP values [2].

In cases suspected for $\mathrm{IIH}$, the radiological finding of transverse sinus stenosis was previously thought to be causative, but recent findings suggest that it is a consequence of the disease $[1,3]$. In clinically persistent patients, transverse sinus stenosis has been managed with endovascular stent therapy [4]. However, in order for this treatment to become routine in clinical practice, its effectiveness should be evaluated in large long-term studies. Contrast-enhanced MRV has been reported to be more reliable than non-contrast flow-based venography in terms of detecting stenosis $[5,6]$. In the current clinical practice, we routinely use contrast-enhanced MRV, if there are no contraindications.

We believe that unilateral transverse sinus hypoplasia, which is frequently encountered in the general population, should not be considered as stenosis. In our patient group, transverse sinus hypoplasia was detected in $20.0 \%$ of patients. Bilateral and focal stenosis, detected in $41.0 \%$ of cases, was used in the radiological scoring system. Stenoses of the sinus lumen due to large Pacchionian granulations were also taken into account.

While IIH can present with many clinical symptoms, loss of vision is most critical. One prospective study reported that the risk of permanent vision loss in the long-term is $4.0 \%$, which requires urgent intervention [7]. In such cases, surgical (optic nerve sheath fenestration, ventriculoperitoneal, and lumboperitoneal shunt) rather than medical or conservative treatments are recommended. In our study, surgery was recommended to one patient, because the complaints did not regress and recurred.

Empty sella was detected in about a half of our patients. Since post-treatment MRI was not performed, there was no information regarding the percentage of empty sella regression. Similarly, other radiological findings also need to be investigated. In one of our cases, post-treatment MRI showed no indication of empty sella, and the pituitary gland became visible (Figure 1). Although empty sella can be observed in the normal population, we believe that in patients with IIH it may be a result of diaphragmatic compression. Notably, automatic measurements were better than manual measurement for an objective assessment the volume of the pituitary volume $[8,15]$. We believe that volumetric evaluation will be too time consuming in the routine clinical setting.
Although high LOP values may indicate IIH, this criterion should be used with caution, because measurement of LOP is performed only at a single time point. The fact that LOP values can vary throughout the day might have prevented us from noticing correlation between LOP and clinical criteria (BMI, vision loss, headache etc.) [9,10]. Moreover, high LOP values have also been reported in the general population [11]. Therefore, it has been proposed that, if diagnosis of IIH doubtful, multiple LOP measurements should be taken [10]. In our study, we did not find any significant correlation between LOP and radiological findings. Based on the available literature and our results, we believe that LOP will be used as a minor criterion in diagnosing IIH in the future.

Few studies have investigated the correlation between combinations of radiographic findings and IIH in children $[12]$ or adults $[13,14]$. These studies demonstrated that combinations of radiographic findings did not significantly contribute to diagnosing IIH. However, these studies did not use contrast-enhanced venography, which is more reliable in showing transverse sinus stenosis. To our knowledge, our study enrolled the highest number of patients who underwent contrast-enhanced MRV imaging for diagnosing IIH.

One of the limitations of our study is a retrospective design. Other limitations include single LOP measurements, differences in the time between MRI and LOP measurements between patients, LOP measurements and papilledema examinations performed by multiple examiners, routine cranial imaging rather than high-resolution imaging with surface coil which is superior in showing orbit findings. Moreover, we did not enroll healthy controls, because of the necessity to use invasive procedures such as contrast-enhanced examination and lumbar puncture.

\section{Conclusions}

In conclusion, no correlation between LOP and reliable radiological features of IIH was observed. This can be due to the variability of LOP values throughout the day, single measurements, and lack of standardization. Prospective studies with pre- and post-treatment MRI and multiple LOP measurements are needed to further investigate and clarify whether there is any correlation between radiological findings and LOP values.

\section{Conflict of interest}

The authors declare that they have no conflict of interest.

\section{References:}

1. Markey KA, Mollan SP, Jensen RH, Sinclair AJ: Understanding idiopathic intracranial hypertension: mechanisms, management, and future directions. Lancet Neurol, 2016; 15: 78-91

2. Friedman DI, Liu GT, Digre KB: Revised diagnostic criteria for the pseudotumor cerebri syndrome in adults and children. Neurology, 2013; 81: 1159-65
3. Baykan B, Ekizoğlu E, Altıokka Uzun G: An update on the pathophysiology of idiopathic intracranial hypertension alias pseudotumor cerebri. Agri, 2015; 27: 63-72

4. Puffer RC, Mustafa W, Lanzino G: Venous sinus stenting for idiopathic intracranial hypertension: A review of the literature. $J$ Neurointerv Surg, 2013; 5: 483-86

5. Nedelmann M, Kaps M, Mueller-Forell W: Venous obstruction and jugular valve insufficiency in idiopathic intracranial hypertension. $\mathrm{J}$ Neurol, 2009; 6: 964-69 
6. Fera F, Bono F, Messina D et al: Comparison of different MR venography techniques for detecting transverse sinus stenosis in idiopathic intracranial hypertension. J Neurol, 2005; 9: 1021-25

7. Wall M, George D: Idiopathic intracranial hypertension. A prospective study of 50 patients. Brain, 1991; 114 (Pt 1A): 155-80

8. Hoffmann J, Schmidt C, Kunte H et al: Volumetric assessment of optic nerve sheath and hypophysis in idiopathic intracranial hypertension. Am J Neuroradiol, 2014; 3: 513-18

9. Johnston I, Paterson A: Benign intracranial hypertension. II. CSF pressure and circulation. Brain, 1974; 2: 301-12

10. Mollan SP, Markey KA, Benzimra JD et al: A practical approach to, diagnosis, assessment and management of idiopathic intracranial hypertension. Pract Neurol, 2014; 6: 380-90
11. Whiteley W, Al-Shahi R, Warlow CP et al: CSF opening pressure: Reference interval and the effect of body mass index. Neurology, 2006; 9: 1690-91

12. Lim MJ, Pushparajah $\mathrm{K}$, Jan $\mathrm{W}$ et al: Magnetic resonance imaging changes in idiopathic intracranial hypertension in children. $\mathrm{J}$ Child Neurol, 2010; 3: 294-99

13. Maralani PJ, Hassanlou M, Torres C et al: Accuracy of brain imaging in the diagnosis of idiopathic intracranial hypertension. Clin Radiol, 2012; 7: 656-63

14. Agid R, Farb RI, Willinsky RA et al: Idiopathic intracranial hypertension: The validity of cross-sectional neuroimaging signs. Neuroradiology, 2006; 8: 521-27

15. Hoffmann J, Huppertz HJ, Schmidt C et al: Morphometric and volumetric MRI changes in idiopathic intracranial hypertension. Cephalalgia, 2013; 13: 1075-84 